\title{
Double fenestrated physician-modified stent-grafts for total aortic arch repair in 50 patients
}

\author{
Lucien Chassin-Trubert, MD, ${ }^{a, b}$ Thomas Gandet, MD, ${ }^{a}$ Youcef Lounes, MD, ${ }^{a}$ Baris Ata Ozdemir, MD, PhD, ${ }^{c}$ \\ Pierre Alric, MD, PhD, ${ }^{a}$ and Ludovic Canaud, MD, PhD, ${ }^{a}$ Montpellier, France; Las Condes, Chile; and Bristol, \\ United Kingdom
}

\begin{abstract}
Objective: Our aim was to evaluate the early- and medium-term outcomes of using double fenestrated physicianmodified endovascular grafts (PMEGs) for total endovascular aortic arch repair.

Methods: The present single-center retrospective analysis of prospectively collected data included 50 patients from January 2017 through October 2019, who had undergone thoracic endovascular aortic repair (TEVAR). The fenestrations were a proximal larger fenestration that incorporated the brachiocephalic trunk and left common carotid artery and a distal smaller fenestration for the left subclavian artery (LSA). Only the LSA fenestration was stented.

Results: The median duration for stent graft modification was $26 \pm 6$ minutes. Of the 50 patients, 41 were men. The mean patient age was $68 \pm 11.5$ years. The indications for treatment included degenerative aortic arch aneurysm ( $\mathrm{n}=17$ ). dissecting aortic arch aneurysm after type A dissection $(n=13)$, type B dissection $(n=13)$, aortic ulcer $(n=3)$, and other pathologies $(n=4)$. The technical success rate was $94 \%$ (47 of 50) overall, and 100\% (28 of 28) after a technical modification incorporating a preloaded guide wire for the LSA fenestration $(P<.05)$. The 30 -day mortality was $2 \%(n=1)$. Two patients (4\%) had a minor stroke with full recovery. One patient (2\%) had a type IB and two patients (4\%) had a type II endoleak from the LSA. Four patients (8\%) required reintervention: one because of a type IB endoleak and three because of access-related complications. All supra-aortic trunks were patent. During a mean follow-up of $16 \pm$ 8.3 months, no conversions to open surgical repair were required and no aortic rupture, paraplegia, or retrograde dissection occurred.

Conclusions: Using double fenestrated PMEGs for TEVAR is both feasible and effective for total endovascular aortic arch repair, avoiding the need for anatomic and extra-anatomic surgical revascularization. The absence of brachiocephalic trunk stenting was not associated with endoleaks or treatment failure and resulted in a lower stroke risk than alternative strategies. The midterm results suggest that stenting of the brachiocephalic trunk and right common carotid artery might not be necessary for a large proportion of patients undergoing total endovascular aortic arch repair. The persistence of the seal and ongoing durability require assessment in studies with long-term follow-up data available.
\end{abstract}

Keywords: Aortic arch; F-TEVAR; Physician-modified stent-graft; TEVAR; Thoracic aorta

Historically, open surgical replacement of the aortic arch was associated with significant risk, with a perioperative mortality rate of $7 \%$ to $12 \%$ reported for elective cases $^{1,2}$ and even greater rates under emergency circumstances. ${ }^{3}$ Significant improvement has occurred in the

\footnotetext{
From the Department of Thoracic and Vascular Surgery, Arnaud de Villeneuve Hospital, Montpellier ${ }^{\mathrm{a}}$; the Servicio de Cirugía Vascular y Endovascular, Clínica Universidad de los Andes, Las Condes ${ }^{\mathrm{b}}$; and the Centre for Surgical Research, Bristol Medical School, University of Bristol. Bristol. ${ }^{\mathrm{C}}$

Author conflict of interest: none.

Additional material for this article may be found online at www.jvascsurg.org. Correspondence: Lucien Chassin-Trubert, MD, Service de Chirurgie Vasculaire et Thoracique, Hôpital A de Villeneuve, 191 av Doyen Gaston Giraud, Montpellier 34090, France (e-mail: info@cirujanovascular.cl).
}

results over time, ${ }^{4,5}$ with the use of various strategies such as selective cerebral perfusion associated with hypothermia during circulatory arrest. ${ }^{6}$ Open surgery of the aortic arch represents the reference standard for pathologies of the aortic arch. However, significant apprehension has continued regarding subjecting a patient to these procedures. This is particularly so for elderly patients, ${ }^{7}$ those requiring emergency repair, and patients with major comorbidities.

In high-risk patients, an alternative is the hybrid procedure with debranching of the supra-aortic vessels. This creates an adequate proximal landing zone for stent-graft repair of the aortic arch. The hybrid procedure still requires sternotomy and clamping of the ascending aorta and supra-aortic branches. An incidence of 30-day mortality of $9 \%$ to $20 \%$ and perioperative stroke of $6.8 \%$ to $14 \%$ have been reported. ${ }^{8-10}$

The recent evolution of stent-graft technology has resulted in the development of custom-made branched stent-grafts to achieve total endovascular aortic arch 
repair. The disadvantages of custom-made devices include the time required for manufacture and delivery for urgent cases and the associated high costs. However, a high rate of embolization is also associated with this approach, probably related to the complexity of deploying a multibranched, unibody, stent-graft (Supplementary Table, online only). ${ }^{17-15}$

A further alternative is the use of physician-modified endografts (PMEGs) for zone $O$ thoracic endovascular aortic repair (TEVAR). Their use involves deployment of a conventional stent-graft device ex vivo and the creation of customized fenestrations and reconstraint into the delivery system. We have previously reported our early experience with 17 patients unfit for open repair and requiring rapid treatment, with good short-term results. ${ }^{16}$ The objectives of the present study were to evaluate the medium-term results for the current series of 50 patients treated with PMEGs for total endovascular aortic repair and compare these findings with the initial learning curve.

\section{METHODS}

Patients. The institutional review board approved the protocol and the written informed consent form for the present study. All the patients gave written informed consent, and the local authorities approved the present study. From January 2017 through October 2019, all patients presenting with aortic arch lesions who had been deemed either unfit for open thoracic surgery or required emergent repair and with suitable anatomy for a double fenestrated PMEG were enrolled and treated at one tertiary referral center (Arnaud de Villeneuve Hospital, Montpellier, France). During the same study period, 85 open surgical procedures of the aortic arch were performed, of which approximately five were frozen elephant trunk procedures.

Multidisciplinary teams, including a cardiovascular surgeon, were involved in the decision making. Patients with zone 2 and zone 1 aortic arch lesions or zone $O$ saccular aneurysms at the lesser curvature of the arch were suitable for the present study, with the full inclusion criteria detailed in Table I. Zone 0 aortic arch lesions, except for saccular aneurysms at the lesser curvature of the arch, were an anatomic exclusion criterion. The demographic, anatomic, intraoperative, and postoperative data were prospectively recorded.

Planning, sizing, and device preparation. The Valiant Captivia stent-graft (Medtronic, Santa Rosa, Calif) was used for all PMEGs. The technical aspects of device modification and fenestration have been previously reported in detail. ${ }^{16}$ A brief summary follows, with incorporation of the most recent modification, a preloaded guidewire. A vascular imaging workstation was used to accurately define and measure the patient's anatomy from computed tomography angiography images. An

\section{ARTICLE HIGHLIGHTS}

Type of Research: A retrospective study of prospectively collected, single-center registry data

Key Findings: Total endovascular aortic repair with double fenestrated physician-modified endovascular grafts for zone 0 thoracic endovascular aortic repair in 50 high surgical risk or emergent patients resulted in $94 \%$ technical success, $2 \%$ in-hospital mortality. $4 \%$ stroke, $0 \%$ type $1 \mathrm{~A}$ endoleak, and $8 \%$ reintervention rates. During $16 \pm 8$ months of follow-up, no patient had died of aortic causes or required secondary intervention and all supra-aortic vessels remained permeable.

Take Home Message: Double fenestrated physicianmodified endovascular grafts are a valid option for zone $\mathrm{O}$ thoracic endovascular aortic repair in highrisk surgical and emergent patients.

appropriate stent graft was selected to achieve the seal and modifications planned. These were a proximal (nearest the leading edge) larger fenestration that incorporated the brachiocephalic trunk (BT) and left common carotid artery (LCCA) and a distal smaller fenestration for the left subclavian artery (LSA). From our early experience, it had become evident that the critical intraprocedural step was successful cannulation of the distal LSA fenestration via a guidewire introduced from the brachial artery. From January 2018 onward, the procedure was refined to include a preloaded guidewire in the stent-graft modifications for the LSA fenestration. To achieve this, the stent-graft must be completely unsheathed on the back table. Fenestrations were fashioned, as previously described. ${ }^{16}$ Subsequently, a needle hole was made in the introducer sheath (graft cover) just distal to the section bearing the endograft, through which a 260-cm, 0.035-in. hydrophilic, stiff, angled guidewire was passed cephalad. The guidewire traversed the inside of the sheath and the deployed endograft before delivery through the LSA fenestration and continued cephalad external to the endograft (Fig 1, A). The endograft was resheathed using nylon tape and snuggers. We avoided embedding or trapping the guidewire in the folds of the graft by keeping the guidewire external to the nylon tape and snuggers. The exit of the guidewire from under the tip of the introducer sheath, in line with the LSA fenestration, is demonstrated in Fig 1, $B$.

Technical steps. All procedures were performed with the patients under general anesthesia using either surgical exposure or percutaneous access of the common femoral arteries and left brachial artery. Systemic heparin of $100 \mathrm{U} / \mathrm{kg}$ was administered after access was obtained, and the activated clotting time was monitored. A 24F, 33- 
Table I. Inclusion and exclusion criteria used for double fenestrated physician-modified endovascular graft for total endovascular aortic arch repair

\begin{tabular}{l}
\hline Criteria \\
\hline Inclusion \\
Proximal and distal neck \\
Length $>20 \mathrm{~mm}$ \\
Diameter $>20$ but $<40 \mathrm{~mm}$ \\
Zone 0 saccular aneurysm at the lesser curvature of the arch \\
Zone 1 aortic arch lesions \\
Zone 2 aortic arch lesions \\
Emergent or elective cases \\
Exclusion \\
Zone O aortic arch lesions, except for saccular aneurysms at \\
lesser curvature of arch
\end{tabular}

$\mathrm{cm}$ length introducer sheath was placed retrograde through the common femoral access. A $7 \mathrm{~F}, 80-\mathrm{cm}$ sheath introducer was placed through the retrograde left brachial access into the ostium of the LSA. A doublecurved, $300-\mathrm{cm}$, extra stiff, 0.035-in. guide wire was positioned against the aortic valve through the femoral access (Supplementary Fig, A, online only). The proximal side of the preloaded guide wire was advanced through the femoral access sheath, delivered to the LSA using a 6F, 25-mm snare loop and exteriorized, establishing a through-and-through wire (Supplementary Fig, B, online only). The PMEG was advanced over the Lunderquist wire (Cook Medical, Bloomington, Ind; Supplementary Fig, C online only), and the preloaded guidewire was progressively pulled by the second operator from the left brachial access, orienting the fenestrations superiorly to face the supra-aortic trunks originating off the superior arch. The first angiographic run (Supplementary Fig, $D$ online only), perpendicular to the LSA, was performed through the left brachial sheath (Supplementary Fig, E, online only). The mean blood pressure was lowered to $\sim 80 \mathrm{~mm} \mathrm{Hg}$, and deployment begun under visualization (Supplementary Fig, F, online only). Rapid pacing was not used. The 7F brachial sheath was advanced with its dilatator through the LSA fenestration over the preloaded guidewire into the stent-graft lumen. An 8- to 12$\mathrm{mm}$ diameter, 38- or 59-mm-long balloon expandable covered stent (Lifestream; Bard, Tempe, Ariz) was inflated (Supplementary Fig, $G$, online only), protruding $\sim 5 \mathrm{~mm}$ into the aortic stent-graft lumen, with the remaining length in the LSA. The covered stent was flared using a 14-mm balloon. Completion angiography was performed to verify the correct position of the PMEGs and patency of all supra-aortic vessel trunks (Supplementary Fig, $H$. online only). The technique of deployment for a double fenestrated PMEG for total endovascular aortic arch repair is summarized in Supplementary Video 1.
Follow-up. Follow-up surveillance was performed using serial computed tomography scans at 1 week, 3, 6, and 12 months, and annually thereafter (Fig 2).

Statistical analysis. Categorical data are presented as frequencies and continuous variables as the median and range or mean \pm standard deviation. Comparisons were performed using the $\chi^{2}$ test for categorical variables and the Student $t$ test for continuous variables. A $P$ value of $<.05$ was considered statistically significant. All statistical analyses were performed using IBM SPSS Statistics 24 software, version 24.0.0.0 (IBM Corp, Armonk, NY).

\section{RESULTS}

From January 2017 through October 2019, 50 patients (41 men; mean age, $68 \pm 11.5$ years; range, 26-87 years) had undergone total endovascular aortic arch repair. Of the 50 procedures, 38 (76\%) were elective. All the elective patients were judged to be unsuitable for open surgical repair because of high surgical risk and associated probability of major complications and mortality. This cohort included patients who would not have been able to tolerate aortic cross-clamping or thoracotomy. Medical comorbidities included significant chronic obstructive pulmonary disease, coronary artery disease, congestive heart failure, and previous thoracic aorta surgery. Details of the demographic data and comorbid conditions are provided in Table II. The indications for total endovascular arch repair were varied and are summarized in Table III.

An average of 1.8 stent-grafts (range, 1-3) were deployed. The mean proximal sealing length was $27.4 \pm 4 \mathrm{~mm}$. The mean length of the proximal fenestration was $26.3 \mathrm{~mm}$ (range, 18-30 mm). The mean length between the fenestrations was $5.3 \mathrm{~mm}$ (range, $5-8 \mathrm{~mm}$ ). The associated procedures included supra-aortic trunk reentry tear closure by covered stent-graft placement in four patients, left carotid-axillary bypass in two patients (unplanned), transcatheter aortic valve replacement in one patient, LSA chimney use in one patient (unplanned), femoral endarterectomy in one patient, femoral-femoral bypass in one patient, and one unplanned iliac conduit to introduce the stent-graft delivery system. The operative details are also provided in Table III.

\section{THIRTY-DAY OUTCOMES}

The median duration for stent-graft modification was $26 \pm 6$ minutes (range, 16-41 minutes). Endovascular exclusion of the aortic arch was achieved in all cases (Supplementary Video 2). In two patients, catheterization of the stent-graft fenestration from the LSA was not achieved, and revascularization was performed using left carotid-axillary bypass. Coverage of the LSA fenestration by additional distal stent-graft placement was used to prevent an endoleak. In one patient, the LSA stent-graft fenestration was misaligned. Having confirmed patency of the BT and LCCA with aortography, 


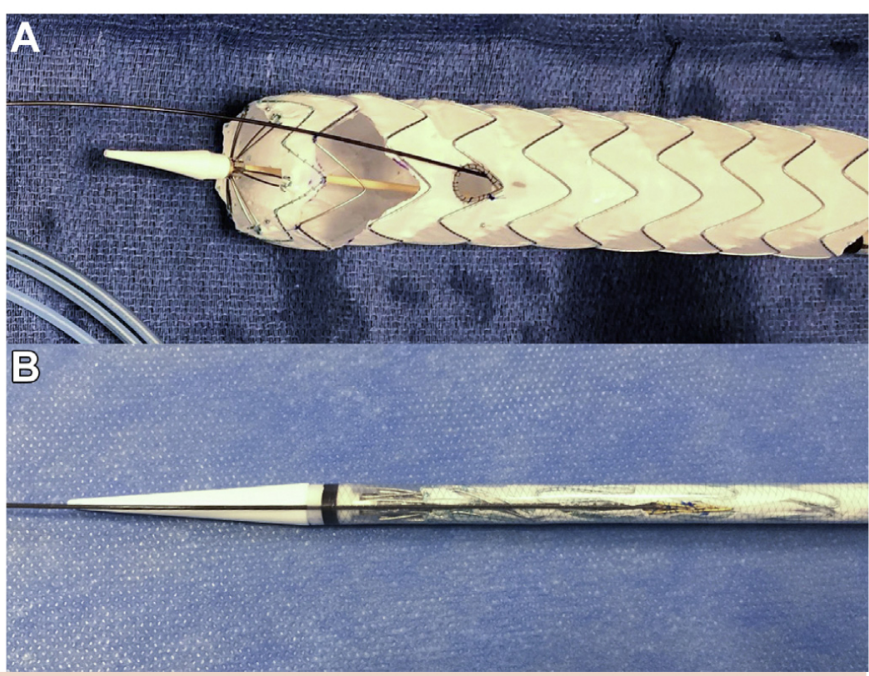

Fig 1. A, The unsheathed custom-made fenestrated Valiant Captivia thoracic stent-graft. The proximal large fenestration for the brachiocephalic trunk (BT) and left common carotid artery (LCCA) is constructed without removing the stent-graft stent struts. The site for the distal circular fenestration for the left subclavian artery (LSA) is selected such that it will not be crossed by any stent struts. A radiopaque marker is included to delineate the fenes tration for the LSA under fluoroscopy. The hydrophilic guidewire is passed cephalad into the sheath through a needle hole and advanced through the stent-graft lumen, exiting through the LSA fenestration and continuing cephalad external to the stent-graft. B, Photograph of stent-graft when resheathed. The guidewire emerges from under the tip of the introducer sheath, in line with the LSA fenestration.

a chimney was used to manage the LSA. We believe that the misalignment was an error of deployment rather than a sizing or modification error. This was the third patient in our series. Two patients (4\%) had experienced a stroke (visual loss and confusion; both with cerebral computed tomography changes). Both had had a full recovery by discharge, with no long-term deficits. The 30day mortality was $2 \%(n=1)$. This patient had died on day 4 after reintervention because of bleeding from the proximal anastomosis of the remnant iliac conduit. Four patients $(8 \%)$ required reintervention. One was for a type IB endoleak, which was successfully treated by deployment of a distal thoracic stent-graft on the third postoperative day. The remaining three patients had required reintervention for access-related complications. Two patients (4\%) had a type II endoleak from the LSA, both without sac expansion. The first was observed and had spontaneously disappeared by the third postoperative month. The second was still under observation at the last follow-up examination. Seven patients (14\%) had presented with access-related complications. All supra-aortic trunks were patent, and no spinal cord ischemia, aortic rupture, perioperative myocardial infarction, retrograde dissection, or conversion to surgical repair had been recorded in the immediate perioperative period.

Failure to achieve cannulation of the LSA fenestration with the left brachial guidewire in three patients triggered the preloaded guidewire modification in the technique from patient 23 onward. In the group of 28 patients $(56 \%)$ with the preloaded LSA guidewire used, the technical success rate was $100 \%$. The outcomes for both groups are further summarized in Table IV.

Follow-up. During a mean follow-up of $16 \pm 8.3$ months, one patient had died at 7 months of pulmonary disease and one patient had died at 10 months of urinary sepsis, for an overall mortality of $6 \%$. No conversions to open surgical repair had been required. Also, no aortic rupture, paraplegia, retrograde dissection, myocardial infarction, graft migration, graft collapse, aorta-related death, or aneurysmal diameter evolution had developed. All supra-aortic trunks were patent.

\section{DISCUSSION}

In the present single-center, retrospective analysis of total endovascular aortic arch repair with double fenestrated PMEGs, no cases of aortic mortality or type IA endoleak had developed, with patency of all supraaortic trunks during a median follow-up of 16 months. The standout feature of this double fenestrated stentgraft modification was the simple manipulation during the procedure. The proximal fenestration is directed to the orifices of the BT and LCCA automatically when the LSA fenestration is catheterized and secured by covered stent placement. Furthermore, because the proximal fenestration is large enough to accommodate the branches with a low risk of branch occlusion, routine stenting of branches is not required. This seems to be crucial to the excellent perioperative outcomes in the present study and contrasts with reports of the use of inner branched stent-grafts in the arch. Although many technical challenges exist specific to the aortic arch for total endovascular arch repair (ie, curved, angulated, short distance to the coronary ostia and aortic valve, hostile hemodynamics, and respiratory motion), during the past decade manufactured branched custom stentgrafts have achieved acceptance. ${ }^{11-15}$ Two custom-made branched devices are currently available: the Cook Abranched arch endograft (third-generation arch design; Cook Medical) and the RelayBranch Thoracic Arch System (Terumo Aortic, Sunrise, Fla). Both devices are intended for hybrid zone O TEVAR, with two inner side branches for the BT and LCCA, but requiring surgical revascularization of the LSA. The worldwide experience with 27 Cook A-branched arch endografts was reported as a multicenter experience in 2016 by Spear et al, ${ }^{12}$ with 30-day mortality, stroke, endoleak, technical success, and secondary procedure rates of $0 \%, 11 \%, 18.5 \%$ 


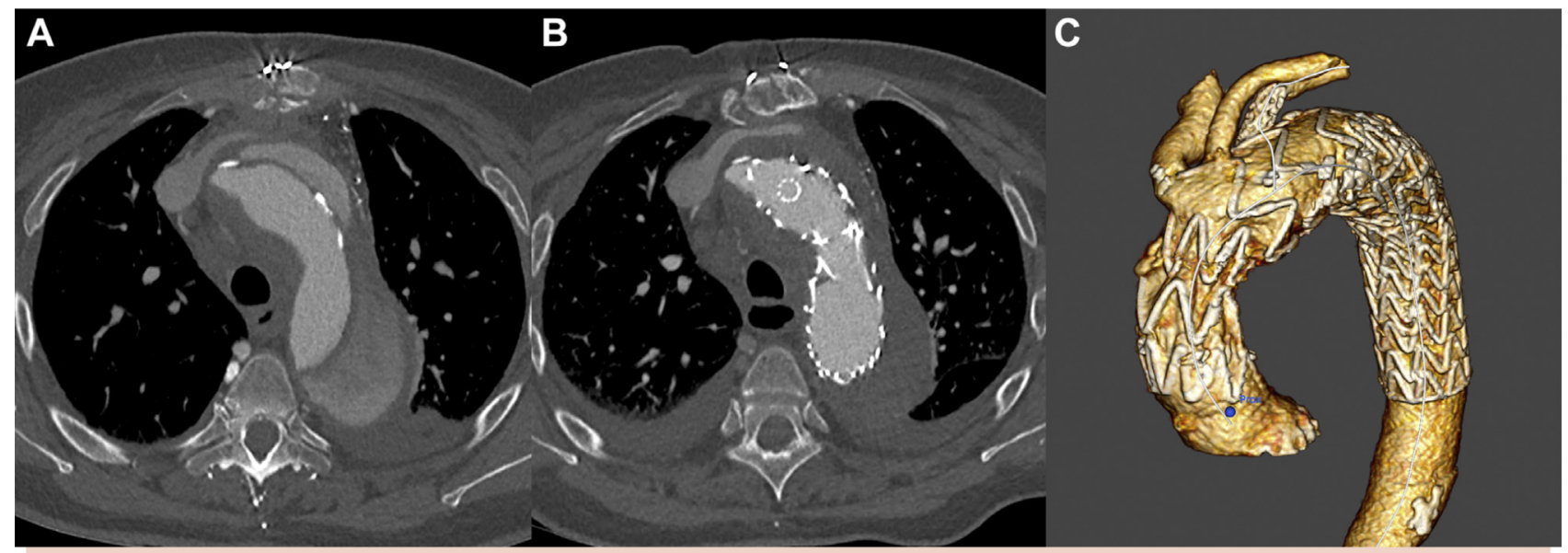

Fig 2. A, Computed tomography scan demonstrating a symptomatic dissecting aortic arch aneurysm after previous surgical treatment of an acute type A dissection. B. Computed tomography scan after double fenestrated physician-modified endovascular graft (PMEG) in zone $O$ treatment demonstrating successful exclusion of the aneurysm. Note the left subclavian artery (LSA) covered stent. C, Three-dimensional volume rendered reconstruction demonstrating the double fenestrated PMEG and patency of the supra-aortic trunks.

$100 \%$, and $22.2 \%$, respectively, at 12 months of follow-up. A more recent study of 54 patients treated with this same custom-made branched stent-graft was reported by Tsilimparis et $\mathrm{al}^{15}$ in 2019 . They reported a 30-day mortality, stroke, endoleak, technical success, and secondary procedure rate of $6 \%, 11 \%, 6 \%, 98 \%$, and $24 \%$, respectively, at 12 months follow-up. ${ }^{15}$ Experience with the RelayBranch Thoracic Arch System was reported in 2019 by Ferrer et $\mathrm{al}^{14}$ in 24 patients, with 30-day mortality, stroke, endoleak, technical success, and secondary procedure rates of $16.6 \%, 25 \%, 8.3 \%, 95.8 \%$, and $16.7 \%$, respectively, at 18 months of follow-up. Despite the experience and skill of the operators, the technical difficulty of side branch catheterization, compounded by the need for surgical revascularization of the LSA, resulted in an inherently high risk of cerebral embolism and other adverse outcomes. In the present series of 50 patients, aligning the graft was based on only the LSA fenestration. This is one of the most important intraoperative advantages of the described approach. The deployment algorithm actively steers the operator away from superfluous manipulations of the device within the arch, guidewire manipulation, and instrumentation and clamping of the BT and LCCA. In addition, no surgical revascularization of the LSA is required. A significantly lower procedural stroke risk was observed. The outcomes with double fenestrated PMEGs for total endovascular aortic repair in the present study seem superior to the reported outcomes available for the two manufactured branched custom stent-grafts, with a 30-day mortality, stroke, endoleak, technical success, and secondary procedure rate of $2 \%, 4 \%, 6 \%, 94 \%$, and $8 \%$, respectively, at 16 months of follow-up.

The desire to stent branches in the aortic arch is driven by concerns regarding achieving a seal and durability. These decisions are extrapolations derived from experience managing visceral branches in the setting of an extensive abdominal or thoracoabdominal aneu rysm, for which the need for stenting of fenestrations is intuitive and clear. The results we have presented suggest that near total stenting of fenestrations is not neces sarily generalizable to fenestrated/branch devices at all anatomic locations. The requirements for seal are determined by the interaction of the anatomy, physiology, and device in the treated zone. Shahcheranhi et al ${ }^{17}$ demonstrated that the higher hemodynamic pressure in the aortic arch is along the outer wall in the vicinity of the supra-aortic branches. In an abdominal aortic aneurysm, the stent-graft is positioned in the middle of the sac, leaving a significant gap between the fenestration and ostium of the target. However, in the aortic arch, once the stent-graft is fully deployed, the hemodynamic forces and angulation will align the deployed stent-graft against the greater curvature or aortic dome, eliminating the gap between the fenestration and vessel ostia. We believe this phenomenon explains the sound results of the described strategy and the absence of endoleaks, despite the use of a bridging covered stent exclusively for the LSA. The use of the LSA bridging stent is only to secure the alignment of the fenestrations and prevent migration over time. Using an unstented fenestration for the BT and LCCA increases the theoretical risk of stent-graft migration and shuttering. However, the stent-graft is anchored by the LSA stent, and migration has not been observed. It is also reassuring that in proximal scalloped TEVAR, no cases of stent-graft migration have been reported. ${ }^{18}$ However, special anatomic features should be considered when using this approach. Zone $\mathrm{O}$ aortic arch lesions, except for saccular aneurysms at the lesser curvature of the arch, are unsuitable. In this context, the size of the proximal fenestration, proximally and laterally larger than that of the BT and LCCA orifice, 
Table II. Demographics and comorbid conditions of patients undergoing total endovascular aortic arch repair $(\mathrm{N}=50)$

\begin{tabular}{|cc|}
\hline Variable & Patients, No. (\%) \\
\hline Male sex & $41(82)$ \\
\hline Mean age, years & 68 \\
\hline Comorbid conditions & \\
\hline Hypertension & $45(90)$ \\
\hline Smoking & $24(48)$ \\
\hline Dyslipidemia & $23(46)$ \\
\hline Previous thoracic aorta surgery & $15(30)$ \\
\hline Coronary disease & $13(26)$ \\
\hline Chronic obstructive pulmonary disease & $11(22)$ \\
\hline Diabetes mellitus & $10(20)$ \\
\hline Renal insufficiency & $8(16)$ \\
\hline Arrhythmia & $7(14)$ \\
\hline Congestive heart failure & $5(10)$ \\
\hline Peripheral arterial disease & $4(8)$ \\
\hline ASA score & \\
\hline 1 & $0(0)$ \\
\hline 2 & $11(22)$ \\
\hline 3 & $34(68)$ \\
\hline 4 & $5(10)$ \\
\hline ASA, American Society of Anesthesiologists. & \\
\hline
\end{tabular}

would be associated with an unacceptably high rate of endoleak.

The described approach is an off-label use of the Valiant Captivia thoracic stent-graft (Medtronic). Concerns regarding the long-term fabric, stent-graft durability, and effect on the general graft ring stability are understandable. In our series, no stent fractures were detected during routine radiologic follow-up examinations. The Montpellier group has previously reported a series of 15 patients with single fenestration PMECs for zone 2 TEVAR with $>3$ years of follow-up, ${ }^{19}$ with no stent fractures or endoleaks, and with all supra-aortic trunk vessels permeable. Further data are needed to establish durability. Careful long-term monitoring and strict surveillance of patients are required to identify complications resulting from potential device failure.

The use of PMECs is a well-established technique and is particularly useful for patients requiring urgent repair because of symptomatic or rapidly enlarging arch lesions who cannot wait 6 to 12 weeks for a patient-specific manufactured device. They are also useful when a patient does not qualify for a custom-made device or when the physician does not have access to a manufactured device because of regulatory issues or cost. However, no quality control is available when making modifications to a device, and their use is outside the instructions for use. The potential exists for inaccuracies in measurements, risk of device contamination, and altered integrity of the device.
Table III. Indications and operative details $(\mathrm{N}=50)$

\begin{tabular}{|c|c|}
\hline Variable & Patients \\
\hline \multicolumn{2}{|l|}{ Operative indication } \\
\hline Degenerative aortic aneurysm & $17(34)$ \\
\hline $\begin{array}{l}\text { Dissecting aortic arch aneurysm } \\
\text { subsequent to type A dissection }\end{array}$ & $13(26)$ \\
\hline $\begin{array}{l}\text { Dissecting aortic arch aneurysm } \\
\text { subsequent to type B dissection }\end{array}$ & $8(16)$ \\
\hline Acute complicated type B dissection & $5(10)$ \\
\hline Penetrating aortic ulcer & $3(6)$ \\
\hline Pseudoaneurysm & $2(4)$ \\
\hline Aortic arch floating thrombus & $1(2)$ \\
\hline $\begin{array}{l}\text { Aberrant right subclavian artery } \\
\text { with Kommerell diverticulum }\end{array}$ & $1(2)$ \\
\hline \multicolumn{2}{|l|}{ Operative details } \\
\hline Elective & $38(76)$ \\
\hline Urgent & $12(24)$ \\
\hline Proximal landing in zone 0 & $49(98)$ \\
\hline Distal landing in zone 4 & $26(52)$ \\
\hline Distal landing in zone 5 & $24(48)$ \\
\hline Proximal stent-graft diameter, $\mathrm{mm}$ & $30-40$ \\
\hline Length of proximal sealing neck, $\mathrm{mm}$ & $27.4 \pm 4$ \\
\hline LSA covered stent diameter, $\mathrm{mm}$ & $8-12$ \\
\hline $\begin{array}{l}\text { Mean back table PMEG preparation time, } \\
\text { minutes }\end{array}$ & $26 \pm 6$ \\
\hline Mean operative time, minutes (range) & $85(45-164)$ \\
\hline \multicolumn{2}{|c|}{$\begin{array}{l}\text { LSA, Left subclavian artery: PMEG, physician-modified endovascular } \\
\text { graft. } \\
\text { Data presented as number (\%), range, mean } \pm \text { standard deviation, or } \\
\text { mean (range). }\end{array}$} \\
\hline
\end{tabular}

The availability of industry-made devices for zone O TEVAR would alleviate concerns regarding physician modification. Although variations of the aortic arch anatomy are numerous and common, the Montpellier group has recently reported an anatomic study, in which they defined and established two areas of the aortic arch from within which all the supra-aortic branches originate in $97 \%$ of patients. These morphologic data, associated with the encouraging outcomes from the present series of 50 patients, suggest that an off-the-shelf double fenestrated thoracic stent-graft is at least conceptually possible. Industry could therefore use this "universal double fenestration pattern" to create a commercially available stent-graft for zone O TEVAR, decreasing costs and increasing availability. The incorporation of a preloaded guidewire increased the reliable execution of the double fenestrated approach.

The Valiant Captivia stent-graft (Medtronic) was used in the present series because of the extensive institutional experience with this device at the study center. The tip capture also facilitated accurate deployment. However, other devices could also be modified, including the Relay Plus (Terumo Aortic) and the Zenith TX2 (Cook Medical). 
Table IV. Patient outcomes stratified by using of preloaded guidewire technique

\begin{tabular}{|c|c|c|c|}
\hline \multirow[b]{2}{*}{ Variable } & \multicolumn{2}{|c|}{ PMEG with preloaded guidewire } & \multirow[b]{2}{*}{$P$ value } \\
\hline & No $(n=22)$ & Yes $(n=28)$ & \\
\hline PMEG preparation time, minutes & $19 \pm 1.1$ & $30 \pm 3.1$ & $<.05$ \\
\hline Operative time, minutes & $98 \pm 7.4$ & $74 \pm 7.7$ & $<.05$ \\
\hline Technical success & $19(86)$ & $28(100)$ & $<.05$ \\
\hline Length of stay, days & $6 \pm 4.5$ & $6 \pm 5.7$ & NA \\
\hline 30-Day mortality & $1(4.5)$ & $\mathrm{O}(0)$ & NA \\
\hline Endoleak & & & NA \\
\hline Type I & $1(4.5)$ & $\mathrm{O}(0)$ & \\
\hline Type II & $1(4.5)$ & $1(3.6)$ & \\
\hline Type III & $\mathrm{O}(0)$ & $\mathrm{O}(0)$ & \\
\hline Cerebrovascular events & $1(4.5)$ & $1(3.6)$ & NA \\
\hline Perioperative myocardial infarction & $\mathrm{O}(0)$ & $\mathrm{O}(0)$ & \\
\hline Retrograde dissection & $\mathrm{O}(0)$ & $\mathrm{O}(0)$ & \\
\hline Aortic rupture & $\mathrm{O}(0)$ & $\mathrm{O}(0)$ & \\
\hline Access-related complication & $3(13.6)$ & $4(14.2)$ & \\
\hline Reintervention & $2(9)$ & $2(7.1)$ & \\
\hline Supra-aortic trunk permeability & $22(100)$ & $28(100)$ & \\
\hline Conversion to open surgical repair & $\mathrm{O}(0)$ & $\mathrm{O}(0)$ & NA \\
\hline Follow-up, months & $25 \pm 5.7$ & $10 \pm 2.8$ & $<.05$ \\
\hline
\end{tabular}

The mechanism of deployment of the Conformable Gore TAG device (W. L. Gore \& Associates, Inc, Flagstaff, Ariz) does not allow reloading the stent graft onto the sheath. We have already successfully tested the new Valiant Navion thoracic stent-graft (Medtronic) on the back table for double fenestrated PMEGs.

Although the technique described is reproducible, the Montpellier group has extensive experience in physician modification of thoracic stent-grafts. Before the total endovascular aortic arch repair series described, the unit gained experience by performing experimental and clinical studies of proximal scalloped PMEGs, ${ }^{20,21}$ followed by single fenestrated PMEGs for zone 2 TEVAR, ${ }^{19}$ and, finally, single fenestrated PMEGs for TEVAR of zone $O$ and zone 1 aortic lesions, combined with cervical debranching procedures. ${ }^{22}$ After the initial experience with the double fenestrated technique, the group studied the incorporation of a preloaded guidewire using bench top cadaveric flow models. The preparation on the back table is longer; however, the total procedural time is significantly decreased. Most importantly, since the introduction of the preloaded guidewire in clinical practice, $100 \%$ technical success has been observed.

With the substantial advances in endovascular techniques and graft materials, we now have several options to achieve an adequate proximal landing zone for zone O TEVAR and preserve supra-aortic flow. Hybrid TEVAR and debranching involves an adjunctive invasive procedure with associated morbidity. The chimney technique is also a potentially minimally invasive strategy. Concerns exist regarding the incidence of type I gutter endoleaks arising from the gap between the chimney graft and main graft. The propensity for endoleaks increases with the number of chimneys included; therefore, in clinical practice, chimney grafts in the thoracic segment are used to manage a single branch. If coverage of two or three supra-aortic branches is required, additional extra-anatomic bypasses are used. Kanaoka et $a^{23}$ recently reported that among the aortic branch reconstruction procedures, the chimney technique was the only maneuver associated with a significant risk of endoleak (odds ratio, 5.3; 95\% confidence interval, 2.3-11.2; $P<$ .001). Alternative strategies include in situ retrograde laser fenestration, which is both feasible and effective for LSA revascularization. ${ }^{24.25}$ Japanese centers have reported extensive experience with the use of three large custom fenestrations for zone O TEVAR, increasing the margin of safety during deployment. Although operative safety is high, large fenestrations, combined with a lack of supra-aortic trunk cannulation (eg, for the LSA) to accurately align with the target vessels, was associated with $32.4 \%$ type I endoleak at discharge and $16.2 \%$ aneurysm enlargement of $>5 \mathrm{~mm}$ at 17 months. ${ }^{26}$

\section{CONCLUSIONS}

The future of aortic arch repair is emerging over the horizon. Until applicable "off-the-shelf" stent-grafts become widely available, the simplicity of deployment with 
limited endovascular maneuvers in the arch, high technical success, low fenestration-related morbidity, and excellent midterm patency support this double fenestrated PMEG technique as a valid option for high-risk patients and patients requiring emergent repair and unable to wait for a custom-made fenestrated or branched device. However, long-term follow-up is necessary to determine the durability of this technique.

\section{AUTHOR CONTRIBUTIONS}

Conception and design: LCT, TG, YL, BO, PA, LC

Analysis and interpretation: LCT, TC, YL, BO, PA, LC

Data collection: LCT, LC

Writing the article: $L C T, T C, Y L, B O, P A, L C$

Critical revision of the article: $L C T, T C, Y L, B O, P A, L C$

Final approval of the article: LCT, TG, YL, BO, PA, LC

Statistical analysis: LCT, LC

Obtained funding: Not applicable

Overall responsibility: LCT

\section{REFERENCES}

1. Halkos ME, Kerendi F, Myung R, Kilgo P. Puskas JD, Chen EP. Selective antegrade cerebral perfusion via right axillary artery cannulation reduces morbidity and mortality after proximal aortic surgery J Thorac Cardiovasc Surg 2009;138:1081-9.

2. Kazui T, Washiyama N. Muhammad BAH. Terada H, Yamashita K, Takinami M, et al. Total arch replacement using aortic arch branched grafts with the aid of antegrade selective cerebral perfusion. Ann Thorac Surg 2000:70:3-8.

3. Kazui T, Washiyama N, Muhammad BAH, Terada H, Yamashita K. Takinami M, et al. Extended total arch replacement for acute type A aortic dissection: experience with seventy patients. J Thorac Cardiovasc Surg 2000:119:558-65.

4. Sundt TM, Orszulak TA, Cook DJ, Schaff HV. Improving results of open arch replacement. Ann Thorac Surg 2008;86:787-96.

5. Strauch JT. Spielvogel D. Lauten A, Galla J. Lansman S, McMurtry K. et al. Technical advances in total aortic arch replacement. Ann Thorac Surg 2004;77:581-90.

6. Kazui T, Yamashita K, Washiyama N, Terada H, Muhammad BAH Susuki T, et al. Usefulness of antegrade selective cerebral perfusion during aortic arch operations. Ann Thorac Surg 2002;74:1806-9.

7. Kurazumi H, Mikamo A, Kudo T, Susuki R, Takahashi M, Shirawasa B, et al. Aortic arch surgery in octogenarians: is it justified? Eur J Cardiothoracic Surg 2014:46:672-7.

8. Canaud L, Hireche K, Berthet J-P, Branchereau P, Marty-Ané C Alric $P$. Endovascular repair of aortic arch lesions in high-risk patients or after previous aortic surgery: midterm results. J Thorac Cardiovasc Surg 2010;140:52-8.

9. Vallejo N, Rodriguez-Lopez JA, Heidari P, Wheatley C, Caparrelli D, Ramaiah V, et al. Hybrid repair of thoracic aortic lesions for zone $\mathrm{O}$ and 1 in high-risk patients. J Vasc Surg 2012;55:318-25.

10. Martin G, Riga C, Gibbs R, Jenkins M. Hamady M, Bicknell C. Shortand long-term results of hybrid arch and proximal descending thoracic aortic repair: a benchmark for new technologies. J Endovasc Ther 2016;23:783-90.
11. Haulon S, Greenberg RK, Spear R, Eagleton M, Abraham C, Lioupis C, et al. Clobal experience with an inner branched arch endograft. J Thorac Cardiovasc Surg 2014:148:1709-16.

12. Spear R, Haulon S, Ohki T, Tsilimparis N, Kanaoka Y, Milne C, et al. Editor's choice - subsequent results for arch aneurysm repair with inner branched endografts. Eur J Vasc Endovasc Surg 2016;51:380-5.

13. Tazaki J, Inoue K. Higami H. Higashitani N. Toma M. Saito N, et al. Thoracic endovascular aortic repair with branched Inoue stent graft for aortic aneurysms. J Vasc Surg 2017;66:1340-8.

14. Ferrer C, Cao P, Coscarella C. Ferri M. Lovato L, Camparini S, et al. iTalian Reglstry of doUble inner branch stent graft for arch PatHology (the TRIUmPH Registry). J Vasc Surg 2019;70:67282.el.

15. Tsilimparis N, Detter C, Law Y, Rohlffs F, Heidenmann F, Brickwedel J, et al. Single-center experience with an inner branched arch endograft. J Vasc Surg 2019;69:977-85.

16. Canaud L, Ozdemir BA, Chassin-Trubert L, Sfeir J, Alric P, Gandet T. Double homemade fenestrated stent graft for total endovascular aortic arch repair. J Vasc Surg 2019;70:1031-8.

17. Shahcheranhi N, Dwyer HA, Cheer AY, Barakat Al, Rutaganira T. Unsteady and three-dimensional simulation of blood flow in the human aortic arch. J Biomech Eng 2002;124:378-87.

18. De Almeida Sandri C. Ribeiro MS. Macedo TA. Vrtiska T. Oderich CS. Planning endovascular aortic repair with standard and fenestratedbranched endografts. J Cardiovasc Surg (Torino) 2017;58:204-17.

19. Chassin-Trubert L. Mandelli M. Ozdemir BA, Alric P. Gandet T. Canaud L. Midterm follow-up of fenestrated and scalloped physician-modified endovascular grafts for zone 2 TEVAR. J Endovasc Ther 2020:377-84.

20. Faure EM, Khantalin I. Peyron PA. Canaud L. Marty-Ané C. Alric P. Experimental assessment of physician modified proximal scalloped stent graft to extend proximal landing zone in the aortic arch. Eur J Vasc Endovasc Surg 2017;54:150-6.

21. Joseph G, Premkumar P. Thomson V. Varghese M. Selvaraj D. Sahajanandan R. Externalized guidewires to facilitate fenestrated endograft deployment in the aortic arch. J Endovasc Ther 2016;23: 160-71.

22. Canaud L, Baba T, Gandet T, Narayama K, Ozdemir B, Shibata T, et al. Physician-modified thoracic stent-grafts for the treatment of aortic arch lesions. J Endovasc Ther 2017:24:542-8.

23. Kanaoka Y, Ohki T, Maeda K, Baba T. Analysis of risk factors for early type I endoleaks after thoracic endovascular aneurysm repair. J Endovasc Ther 2017:24:89-96.

24. Redlinger RE, Ahanchi SS, Panneton JM. In situ laser fenestration during emergent thoracic endovascular aortic repair is an effective method for left subclavian artery revascularization. J Vasc Surg 2013:58:1171-7.

25. Qin J, Zhao Z, Wang R, Ye K, Li W, Liu X, et al. In situ laser fenestration is a feasible method for revascularization of aortic arch during thoracic endovascular aortic repair. J Am Heart Assoc 2017:6:1-11.

26. Kurimoto Y, Maruyama R, Ujihira K, Nishioka N, Hasegawa K, Iba Y, et al. Thoracic endovascular aortic repair for challenging aortic arch diseases using fenestrated stent grafts from zone O. Ann Thorac Surg 2015:100:24-33. 
Supplementary Table (online only). Reported series of patients treated with custom-made branched stent-grafts for zone O thoracic endovascular aortic repair

\begin{tabular}{|c|c|c|c|c|c|c|c|}
\hline Investigator & Stent-Graft & Patients, No. & $\begin{array}{l}\text { Technical } \\
\text { success, \% }\end{array}$ & $\begin{array}{c}\text { 30-Day } \\
\text { mortality, \% }\end{array}$ & Stroke, \% & Endoleak, \% & $\begin{array}{l}\text { Follow-up } \\
\text { months }\end{array}$ \\
\hline Haulon et al, 2014 & $\begin{array}{c}\text { Cook } A \text { branched } \\
\text { arch endograft }\end{array}$ & 38 & 84 & 13.2 & 15.8 & 36.8 & 12 \\
\hline Spear et al, ${ }^{12} 2016$ & $\begin{array}{c}\text { Cook } A \text { branched } \\
\text { arch endograft }\end{array}$ & 27 & 100 & 0 & 11.1 & 18.5 & 12 \\
\hline Tazaki et al, ${ }^{13} 2017$ & $\begin{array}{l}\text { Branched Inoue stent } \\
\text { graft }\end{array}$ & 7 & 100 & 29 & 42 & 0 & NA \\
\hline Ferrer et al, ${ }^{14} 2019$ & $\begin{array}{l}\text { RelayBranch thoracic } \\
\text { arch }\end{array}$ & 24 & 95.8 & 16.6 & 25 & 8.3 & 18 \\
\hline Tsilimparis et al. ${ }^{15} 2019$ & $\begin{array}{c}\text { Cook } A \text { branched } \\
\text { arch endograft }\end{array}$ & 54 & 98 & 6 & 11 & 6 & 12 \\
\hline
\end{tabular}

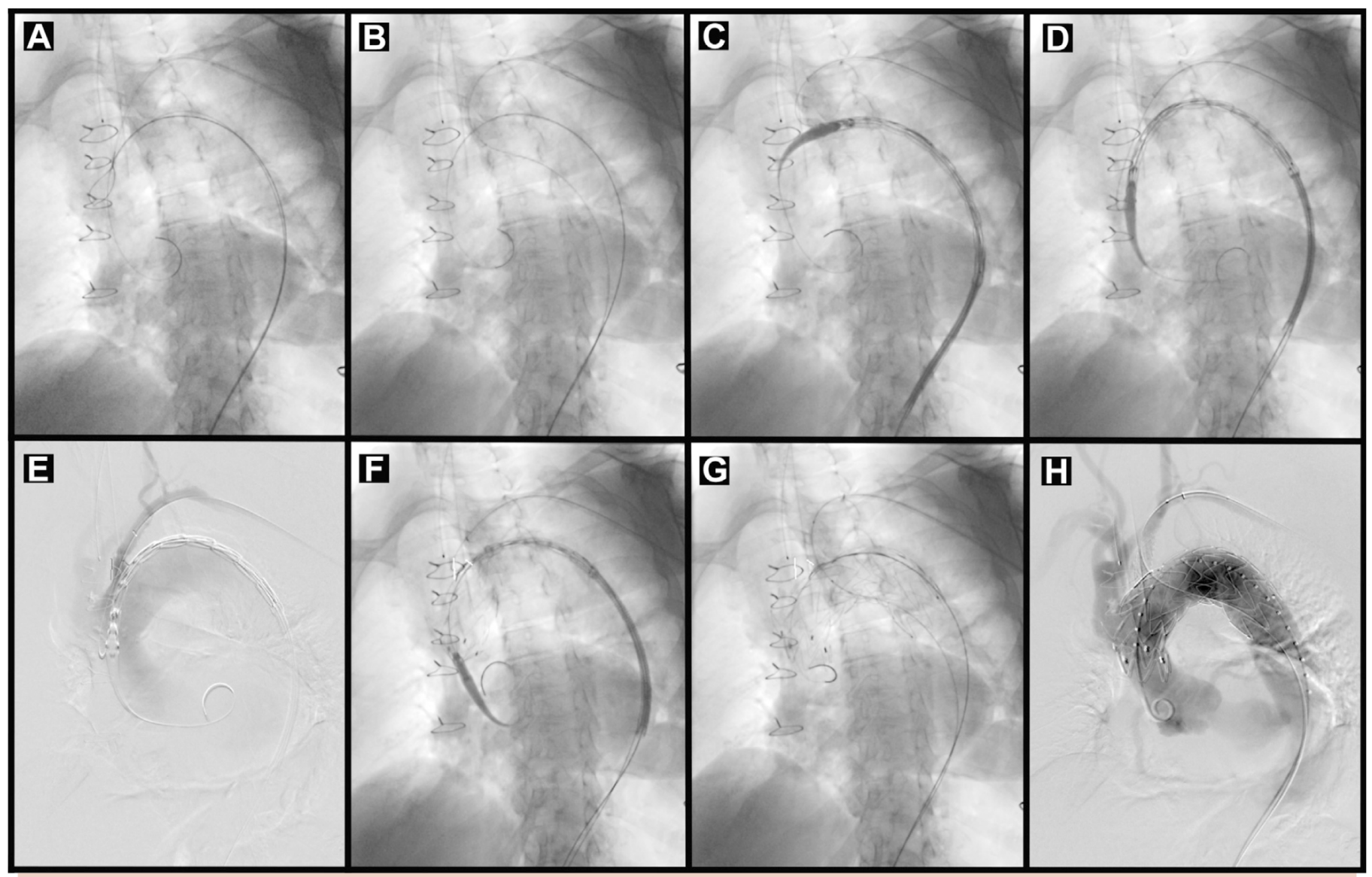

Supplementary Fig (online only). Imaging studies of the technique of deployment for double fenestrated physician-modified endovascular grafts (PMEGs) for total endovascular aortic repair. A, A 7F, 80-cm sheath positioned retrograde from left brachial access into the ostium of the left subclavian artery (LSA). A 0.035-in. double-curved extrastiff, $300-\mathrm{cm}$ wire was positioned against the aortic valve from the femoral access. B. The proximal end of the preloaded guide wire was advanced through the femoral access sheath and delivered to the LSA using a 6F, 25-mm snare loop introduced from the LSA. C. A PMEG was advanced over the Lunderquist wire. D, A PMEG was positioned in the aortic arch. E, Angiography via the left brachial sheath. F, After ascertaining that the LSA fenestration was oriented toward the supra-aortic trunk target vessel, the mean blood pressure was lowered to $\sim 80 \mathrm{~mm} \mathrm{Hg}$, and PMEG was deployed under direct fluoroscopic visualization. G, A balloon expandable covered stent was inflated in the LSA and protruding into the aortic stent-graft lumen. $\mathbf{H}$, Completion angiography was performed to verify the correct position of the PMEC and exclude other potential complications, with patency of all supra-aortic vessel trunks. 